\title{
Defending Grey Attacks by Exploiting Wavelet Analysis in Collaborative Filtering Recommender Systems
}

\author{
Zhihai Yang, Zhongmin Cai ${ }^{*}$ and Aghil Esmaeilikelishomi \\ Ministry of Education Key Lab for Intelligent Networks and Network Security, \\ Xi'an Jiaotong University, Xi'an, 710049, China
}

\begin{abstract}
Shilling" attacks or "profile injection" attacks have always major challenges in collaborative filtering recommender systems (CFRSs). Many efforts have been devoted to improve collaborative filtering techniques which can eliminate the "shilling" attacks. However, most of them focused on detecting push attack or nuke attack which is rated with the highest score or lowest score on the target items. Few pay attention to grey attack when a target item is rated with a lower or higher scores than the average score, which shows a more hidden rating behavior than push or nuke attack. In this paper, we present a novel detection method to make recommender systems resistant to such attacks. To characterize grey ratings, we exploit rating deviation of item to discriminate between grey attack profiles and genuine profiles. In addition, we also employ novelty and popularity of item to construct rating series. Since it is difficult to discriminate between the rating series of attacker and genuine users, we incorporate into discrete wavelet transform (DWT) to amplify these differences based on the rating series of rating deviation, novelty and popularity, respectively. Finally, we respectively extract features from rating series of rating deviation-based, novelty-based and popularitybased by using amplitude domain analysis method and combine all clustered results as our detection results. We conduct a list of experiments on the Book-Crossing dataset in diverse attack models. Experimental results were included to validate the effectiveness of our approach in comparison with benchmarked methods.
\end{abstract}

Keywords-recommender system; grey attack; discrete wavelet transform; shilling attack

\section{INTRODUCTION}

Collaborative filtering recommender systems (CFRSs) have become a popular and effective tool for information retrieval especially when users facing information overload. CFRSs also have played an important role in many popular web services such as Netflix, Amazon etc., which are designed to recommend items based on relevant information for the specific user [3], [5], [11], [14], [30], [33]. However, CFRSs are particularly vulnerable to "shilling" attacks or "profile injection" attacks in which an attacker signs up as a number of "puppet" users and rates fake scores in an attempt to promote or demote the recommendations of specific items by using knowledge of the recommender algorithms [2], [20], [21], [25], [26]. In such attacks, the attackers deliberately insert attack profiles into genuine profiles to change the prediction results which would reduce the trustworthiness of recommendation.
The attack profiles indicate the attacker's intention that he wishes a particular item can be rated highest score (called push attack) or lowest score (called nuke attack) [4], [6], [7], [9], [10], [12], [16], [18], [19]. In addition, to avoid being detected easily by traditional detection techniques, the attackers may rate a higher score or lower score on the target items, which generates relatively hidden attack intents in comparison with push attacks or nuke attacks [24], we also call them grey attacks. Of course, they belong to the "shilling" attacks. Therefore, constructing an effective system to defend the attackers and remove them from the CFRSs is crucial.

Although existing work in this area have focused on detecting and preventing the "shilling" attacks or "profile injection" attacks, it has not reached a fully acceptable level of detection performance. We can briefly summarize that it is difficult to improve detection performance for detecting such attacks when filler size or attack size is small. Moreover, few pay attention to the grey attack detection. As an attacker demotes (nuke attack) the target items by rating lowest score or promotes (push attack) the target items by rating highest score, he also can demote or promote the target items by rating lower or higher scores. In fact, the rating behavior of an attacker is very similar to the behavior of a genuine user if the rating of target item is close to the actual rating. For the nuke attack, an attacker is simply shifting the rating given to the target item from the minimum rating to a rating one step higher, for the push attack, and vice versa [24]. Any profile that includes these ratings is likely to be less suspect. Although a minor change, this has a key effect. Thus, a challenging detection method should not only perform well when attack size or filler size is small, but also effectively defend the grey attack profiles.

In this paper, we propose an unsupervised attack detection method to make recommender systems resistant to such attacks, which combines discrete wavelet transform (DWT) and EM-based (Expectation-maximization based) clustering method. Since the attackers mimic some rating details of genuine users in shilling attacks, the rating behavior between attackers and genuine users will become more similar, especially for the grey attacks. Although existing features extracted from user profiles can characterize the shilling attacks to some extent, it's difficult to fully discriminate between attack profiles and genuine profiles. Moreover, the above challenges are also significant in grey attacks. Our basic assumption is that we can use DWT to amplify the differences between attack profiles and genuine profiles. In addition, to 
characterize the features of grey ratings, we use rating deviation of item to address this crucial problem. To construct input series for DWT, we create a list of transformed rating series to address this problem, which exploits the novelty, popularity and rating deviation of item for each user profiles, respectively. Moreover, we employ the empirical model decomposition (EMD) method to extract intrinsic mode functions (IMFs) from the rating series [17]. These can be seen that there are some but not obvious difference between the attack profiles and genuine profiles (as shown in Figures 4-6). To amplify the difference, we further use DWT to transform these series. In essence, a rating series is a non-stationary random series. Therefore, it is very suitable to be processed by DWT which performs well for non-stationary data [17]. After DWT, the differences between attack profiles and genuine profiles become more obvious (as shown in Figures 7-9). Based on the output series of DWT, we extract a list of effective features by using amplitude domain analysis method. And then exploiting EM clustering method to discriminate jointly attackers and genuine users based on the extracted features. In addition, the effectiveness of our proposed approach is validated and benchmark methods are briefly discussed. Experimental results show that our approach performs well for detecting the grey attacks in comparison with the benchmarked methods.

The remaining parts of this paper are organized as follows: Section 2 reviews some related work. Section 3 describes the attack model and introduces the theory of discrete wavelet transform. Our proposed detection method is introduced in Section 4. Experimental results and analysis are presented and discussed in Section 5. Finally, we conclude the paper with a brief summary and directions for future work.

\section{RELATED WORK}

Although existing detection techniques have focused on detecting and preventing the "shilling" attacks or "profile injection" attacks, it has not reached a fully acceptable level of detection performance. To name only a few, Burke et al. [3] proposed and studied several attributes derived from user profiles for their utility in attack detection. They employed $\mathrm{kNN}$ as their classification approach. However, it was unsuccessful when detecting attacks with small filler size and also suffered from low classifier precision. Then, Williams et al. [15], [24], [28] used several trained classifiers to detect shilling attacks based on extracted features of user profiles. Although, [24] used the higher or lower ratings instead of the maximum or minimum ratings to the target item, discussion of detecting such attacks was limited. Moreover, the detection performance was limited when filler size is small. Mobasher et al. [29] employed signatures of attack profiles and were moderately accurate. But, the method suffered from low accuracy in detecting shilling attack. They just focused on individual users and mostly ignored the combined effect of such attackers. In addition, the detection performance was limited when the attack profiles are obfuscated. Zhang et al. [31] proposed an ensemble approach to improve the precision of detection by using meta-learning technique. Their proposed method performs better detection performance than the bench marked methods. He et al. [32] employed rough set theory to detect shilling attacks though taking features of user profiles as the condition attributes of the decision table. However, their method also suffered from low precision. F. Zhang et al. [17] proposed an online method to detect profile injection attacks based on HHT and SMV. Zhou et al. [1] proposed a detection technique for identifying group attack profiles, called DeRTIA, which combines an improved metric based on Degree of Similarity with Top Neighbors (DegSim) and Rating Deviation from Mean Agreement (RDMA). Zhang et al. [19] proposed a spectral clustering method to make recommender systems resistant to the shilling attacks in the case that the attack profiles are highly correlated with each other. Their experimental results reported good performance in random, average and bandwagon attacks. However, it also performed poor precision and recall in AOP attack when attack size is small.

\section{PRELIMINARIES}

In this section, we firstly describe the attack profiles and attack models. Then, we introduce the theory of discrete wavelet transform to facilitate discussions later.

\section{A. Attack profiles and attack models}

In the literature, "shilling" attacks are classified into two ways: nuke attack and push attack [3]. In order to nuke or push a target item, the attacker should be clearly known the form of an attack profile. The general form of an attack profile is shown in Table 1. The details of the four sets of items are described as follows:

$\mathrm{I}_{\mathrm{S}}$ : The set of selected items with specified rating by the function $\sigma\left(\mathrm{i}_{\mathrm{k}}^{\mathrm{S}}\right)[13]$;

$\mathrm{I}_{\mathrm{F}}$ : A set of filler items, received items with randomly chosen by the function $\rho\left(\mathrm{i}_{1}^{\mathrm{F}}\right)$;

\section{$\mathrm{I}_{\mathrm{N}}$ : A set of items with no ratings;}

$\mathrm{I}_{\mathrm{T}}$ : A set of target items with singleton or multiple items, called single-target attack or multiple-targets attack. The rating is $\gamma\left(\mathrm{i}_{\mathrm{j}}^{\mathrm{T}}\right)$, generally rated the maximum or minimum value in the entire profiles.

In this paper, we utilize 8 attack models to generate attack profiles. The involved attack profiles and corresponding explanations are listed in Table 2. The details of these attack models in our experiments are described as follows:

1) AOP attack: A simple and effective strategy to obfuscate the Average attack is to choose filler items with equal probability from the top $x \%$ of most popular items rather than from the entire collection of items [22].

2) Random attack: $I_{S}=\emptyset$ and $\rho(i) \sim N\left(\bar{r}, \bar{\sigma}^{2}\right)[13]$;

3) Average attack: $I_{S}=\emptyset$ and $\rho(i) \sim N\left(\bar{r}_{i}, \bar{\sigma}_{i}^{2}\right)$ [13];

4) Bandwagon (average): $I_{S}$ contains a set of popular items. Then, we use these items as $I_{S}$, $\sigma(i)=r_{\max }$ or $r_{\min }$ or $r_{\text {grey }}$ (push or nuke or grey) and $\rho(i) \sim N\left(\bar{r}_{i}, \bar{\sigma}_{i}^{2}\right)[13]$;

5) Bandwagon (random): $I_{S}$ contains a set of popular items, 
TABLE I. General Form OF ATtack ProfiLES

\begin{tabular}{|c|c|c|c|c|c|c|c|c|c|c|c|}
\hline \multicolumn{3}{|c|}{$\mathbf{I}_{\mathrm{T}}$} & \multicolumn{3}{|c|}{$\mathbf{I}_{\boldsymbol{S}}$} & \multicolumn{3}{|c|}{$\mathbf{I}_{\mathrm{F}}$} & \multicolumn{3}{|c|}{$\mathbf{I}_{\boldsymbol{N}}$} \\
\hline $\boldsymbol{i}_{1}^{T}$ & $\ldots$ & $\boldsymbol{i}_{j}^{T}$ & $\boldsymbol{i}_{1}^{S}$ & $\ldots$ & $\boldsymbol{i}_{\boldsymbol{k}}^{\boldsymbol{S}}$ & $\boldsymbol{i}_{\mathbf{1}}^{\boldsymbol{T}}$ & $\ldots$ & $\boldsymbol{i}_{\boldsymbol{l}}^{\boldsymbol{F}}$ & $\boldsymbol{i}_{\boldsymbol{1}}^{N}$ & $\ldots$ & $\boldsymbol{i}_{v}^{N}$ \\
\hline$\gamma\left(\mathrm{i}_{1}^{\mathrm{T}}\right)$ & $\ldots$ & $\gamma\left(\mathrm{i}_{\mathrm{j}}^{\mathrm{T}}\right)$ & $\sigma\left(\mathrm{i}_{1}^{\mathrm{S}}\right)$ & $\ldots$ & $\sigma\left(\mathrm{i}_{\mathrm{k}}^{\mathrm{S}}\right)$ & $\rho\left(\mathrm{i}_{1}^{\mathrm{F}}\right)$ & $\ldots$ & $\rho\left(\mathrm{i}_{1}^{\mathrm{F}}\right)$ & null & $\ldots$ & null \\
\hline
\end{tabular}

TABLE II. ATTACK MODELS

\begin{tabular}{|c|c|c|c|c|c|c|}
\hline \multirow{2}{*}{ Attack Model } & \multicolumn{2}{|l|}{$\mathbf{I}_{\mathbf{S}}$} & \multicolumn{2}{|c|}{$\mathbf{I}_{\mathbf{F}}$} & \multirow{2}{*}{$\mathbf{I}_{\mathbf{N}}$} & \multirow{2}{*}{$\begin{array}{c}\mathbf{I}_{\mathrm{T}} \\
\text { (push or nuke or grey) }\end{array}$} \\
\hline & Items & Rating & Items & Rating & & \\
\hline AOP & \multicolumn{2}{|c|}{ null } & \multicolumn{2}{|c|}{$\mathrm{x}-\%$ popular items, ratings set with normal dist around item mean. } & null & $r_{\max }$ or $r_{\min }$ or $r_{\text {grey }}$ \\
\hline Random & \multicolumn{2}{|c|}{ null } & randomly chosen & system mean & null & $r_{\max }$ or $r_{\min }$ or $r_{\text {grey }}$ \\
\hline Average & \multicolumn{2}{|c|}{ null } & randomly chosen & item mean & null & $r_{\max }$ or $r_{\min }$ or $r_{\text {grey }}$ \\
\hline Bandwago (average) & popular items & $r_{\max }$ or $r_{\min }$ & randomly chosen & item mean & null & $r_{\max }$ or $r_{\min }$ or $r_{\text {grey }}$ \\
\hline Bandwagon (random) & popular items & $r_{\max }$ or $r_{\min }$ & randomly chosen & system mean & null & $r_{\max }$ or $r_{\min }$ or $r_{\text {grey }}$ \\
\hline Segment & segmented items & $r_{\max }$ or $r_{\min }$ & randomly chosen & $\mathrm{r}_{\min }$ or $\mathrm{r}_{\max }$ & null & $r_{\max }$ or $r_{\min }$ or $r_{\text {grey }}$ \\
\hline Reverse Bandwagon & unpopular items & $r_{\min }$ or $r_{\max }$ & randomly chosen & system mean & null & $r_{\max }$ or $r_{\min }$ or $r_{\text {grey }}$ \\
\hline Love/Hate & null & null & randomly chosen & $r_{\min }$ or $r_{\max }$ & null & $r_{\max }$ or $r_{\min }$ or $r_{\text {grey }}$ \\
\hline
\end{tabular}

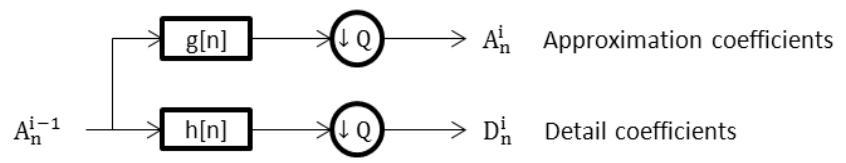

Fig. 1. Block diagram of filter analysis

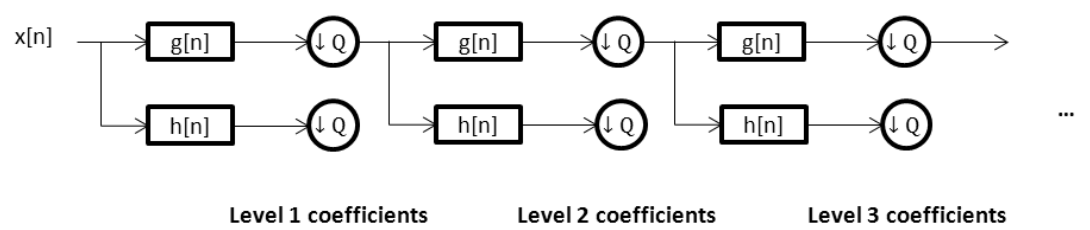

Fig. 2. $\mathrm{K}$ (k greater than or equal to 1$)$ levels of filter bank

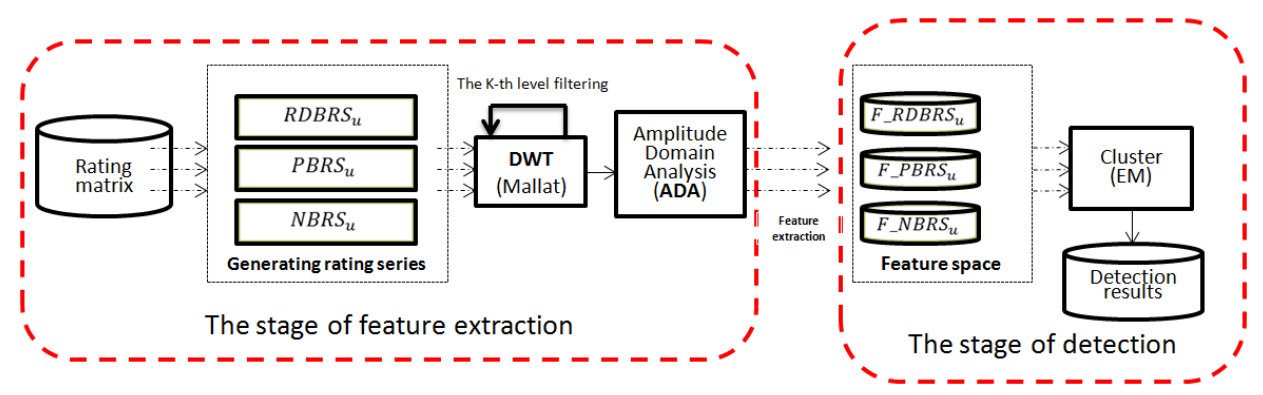

Fig. 3. The framework of our proposed method which consists of two stages: the stage of feature extraction and the stage of detection

$\sigma(i)=r_{\text {max }}$ or $r_{\text {min }}$ or $r_{\text {grey }}$ and $\rho(i) \sim N\left(\bar{r}, \bar{\sigma}^{2}\right)$ (nuke or grey) [13];

6) Segment attack: $I_{S}$ contains a set of segmented items, $\sigma(i)=r_{\max }$ or $r_{\min }$ or $r_{\text {grey }}$ $\rho(i)=r_{\min }$ or $r_{\max }$ or $r_{\text {grey }}$ (push or nuke or grey) [8];

7) Reverse Bandwagon attack: $I_{S}$ contains a set of unpopular items, $\sigma(i)=r_{\min }$ or $r_{\max }$ or $r_{\text {grey }}$ (push or nuke or grey) and $\rho(i) \sim N\left(\bar{r}, \bar{\sigma}^{2}\right)[9]$;

8) Love/Hate attack: $I_{S}=\emptyset$ and $\rho(i)=r_{\text {max }}$ or $r_{\text {grey }}$ (nuke or grey) [9].

\section{B. Discrete wavelet transform}

Discrete wavelet transform (DWT) has been recognized as a natural wavelet transform for discrete time signals. Both time and scale parameters are discrete. For a discrete-time sequence $x[n], n \in Z$, DWT is defined by discrete-time multiresolution decomposition which could be computed by Mallat pyramidal decomposition algorithm (as shown in Equations (1)-(3)) [23]. However, since half the frequencies of the signal have now been removed, half the samples can be discarded according to Nyquist's rule. The filter outputs are then sub- 
sampled by 2 (Mallat's and the common notation is the opposite, g- high pass and h- low pass):

$$
\begin{gathered}
A_{n}^{0}=x[n], n \in N \\
A_{n}^{i}=\sum_{k \in Z} g(k-2 n) A_{k}^{i-1}, i=1,2, \ldots, L \\
\quad D_{n}^{i}=\sum_{k \in Z} h(k-2 n) A_{k}^{i-1}, i=1,2, \ldots,
\end{gathered}
$$

where $h$ and $g$ are impulse responses of high-pass filter $H$ and low-pass filter $G$, respectively. $\left\{A_{n}^{i}\right\}$ and $\left\{D_{n}^{i}\right\}$ are scale sequence and wavelet sequence of $2^{-i}$ scale. $L$ is the maximum possible scale of the discrete signal $x[n]$. The signal is also decomposed simultaneously using a high-pass filter. The outputs give the detail coefficients (from the high-pass filter) and approximation coefficients (from the low-pass) as shown in Figure 1. It is important that the two filters are related to each other and they are known as a quartered mirror filter.

DWT of a signal is calculated by passing it through a series of filters. The decomposition is repeated to further increase the frequency resolution and the approximation coefficients decomposed with high and low pass filters and then downsampled (see Figure 2). This is represented as a binary tree with nodes representing a sub-space with different timefrequency localization. And the tree is known as a filter bank.

\section{OUR PROPOSED APPROACH}

In this section, we firstly introduce the framework of our proposed approach. And then we give several definitions of rating series used in this paper. Finally, we briefly describe our detection method.

\section{A. The framework}

As shown in Figure 3, our proposed algorithm consists of two stages: the stage of feature extraction and the stage of detection. At the stage of feature extraction, the feature is extracted one by one from user profiles by using the proposed feature extraction method (see subsection 4.2). Inspired from previous studies (Zhang et al. [17]), we incorporate into two concepts: Empirical Mode Decomposition (EMD) and Intrinsic Mode function (IMF). EMD is an adaptive and highly efficient decomposition method and is also a necessary step to reduce any given data into a collection of intrinsic mode functions (IMF) where the DWT analysis can be applied. As we all know, DWT is a method for analyzing non-stationary data, since the rating series are non-stationary data. The IMF is defined as a function that satisfies the following requirements: (a) In the whole data set, the number of extreme and zerocrossings must either be equal or differ at most by one; (b) At any point, the mean value of the envelope defined by the local maxima and the envelope defined by the local minima is zero.

With this method, rating series can be decomposed into a finite signal and regard the signal as the input of discrete wavelet transform [17], [27]. In our proposed approach, we decompose respectively each user profiles into novelty-based, popularity-based and rating deviation-based rating series as the input signals. And then, the input signals are passed through the series of filters (including low-pass and high-pass filter, as shown in Figure 3.) to generate corresponding output signals. In the process of DWT, we perform one level transformation to get the output signals. Then, by using amplitude domain analysis method to extract features from the output signal. At the stage of detection, based on the extracted features, we respectively use EM method to cluster two groups. Finally, combing the three parts of clustering results to return our detection result.

\section{B. Feature extraction}

Previous studies [17] have disclosed that using the novelty and popularity of items to construct rating series for user profiles implies useful information. Inspired from this research, we investigate using rating deviation of items to construct rating series in order to extract features from grey attack profiles. Novelty ${ }^{1}$ in recommendation is focusing on recommending the log-tail items (i.e., less popular items) which is generally considered to be particularly valuable to users. Popularity of items usually reflects the genuine users' tastes or preferences in collaborative recommender system. By sorting the items according to their novelty, popularity and rating deviation, we can create respectively the rating deviation-based, novelty-based and popularity-based rating series for the user profiles. Firstly, two definitions of the rating deviation are described in the following:

\section{Definition 1 (Rating Deviation of Items, RDoI).}

The RDoI $\mathrm{I}_{\mathrm{i}}$ (rating deviation of item $\mathrm{i}$ ) is defined as follows:

$$
\mathrm{RDoI}_{\mathrm{i}}=\left\{\begin{array}{cl}
\left|\mathrm{r}_{\mathrm{ui}}-\overline{\mathrm{r}_{\mathrm{i}}}\right|, & \mathrm{r}_{\mathrm{ui}} \neq \perp, \mathrm{u} \in \mathrm{R}_{\mathrm{g}}, \\
0, & \mathrm{r}_{\mathrm{ui}}=\perp
\end{array}\right.
$$

where $r_{u i}$ denotes the rating of user $u$ on item $i . \overline{r_{1}}$ is the mean rating of item $i$ in the system. $r_{u i} \neq \perp$ denotes item $i$ is rated by user $\mathrm{u}, \mathrm{r}_{\mathrm{ui}}=\perp$ denotes item $\mathrm{i}$ is not rated by user $\mathrm{u}$. $\mathrm{R}_{\mathrm{g}}$ denotes the set of genuine users in dataset.

Definition 2 (Rating Deviation-based Rating Series, RDBRS).

Let $\mathrm{RDoI}_{\mathrm{i}}$ denotes the rating deviation of item i. Sort all items in set $I$ (a set of the entire items in the recommender system.) according to $\mathrm{RDoI}_{\mathrm{i}}$ in descending order and let $i=1,2, \ldots,|I|$ denotes the order of items after sorting, where |I| denotes total number of items in the recommender system. The $\operatorname{RDBRS}_{\mathrm{u}}(\mathrm{i})^{2}$ is defined as follows:

$$
\begin{aligned}
& \operatorname{RDBRS}_{\mathrm{u}}(\mathrm{i})= \\
& \left\{\begin{array}{cl}
1, & \mathrm{r}_{\mathrm{u}, \mathrm{i}} \neq \perp \text { and }\left(\mathrm{i}=1 \text { or } \operatorname{RDNRS}_{\mathrm{u}}(\mathrm{i}-1) \neq 1\right), \\
-1, & \mathrm{r}_{\mathrm{u}, \mathrm{i}}=\perp \text { and }\left(\mathrm{i}=1 \text { or } \operatorname{RDNRS}_{\mathrm{u}}(\mathrm{i}-1) \neq-1\right), \\
0, & \text { otherwise. }
\end{array}\right.
\end{aligned}
$$

where zero value is used to meet the requirements of extreme for DWT. $r_{u, i} \neq \perp$ denotes item $i$ is rated by user $u$. $r_{u, i}=\perp$ denotes item $\mathrm{i}$ is not rated by user $\mathrm{u}$.

\section{Novelty of Items, NoI}

The $\mathrm{NoI}_{\mathrm{i}}$ (novelty of item $\mathrm{i}$ ) is defined as follows:

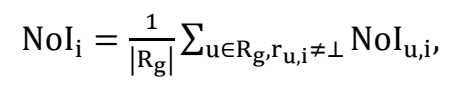

\footnotetext{
${ }^{1}$ The novelty of an item refers to the degree to which it is unusual with respect to the user's normal tastes.

2 The rating deviation-based rating series of user $u$.
} 
where $\mathrm{NoI}_{\mathrm{u}, \mathrm{i}}$ denotes the novelty of item $i$ for user $\mathrm{u}$ [17].

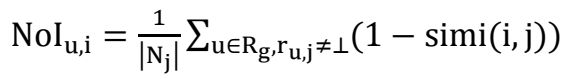

where $\mathrm{N}_{\mathrm{j}}$ denotes the number of users who rate on item $\mathrm{j}$. $R_{g}$ denotes the set of genuine users in dataset. $\operatorname{simi}(i, j)$
(Jaccard coefficient) denotes the similarity between item $i$ and item $j$, which can be calculated as follows:

$$
\operatorname{simi}(i, j)=\frac{\left|V_{i} \cap V_{j}\right|}{\left|V_{i} \cup V_{j}\right|}
$$

Where $V_{i}$ is set of users rated by item $i, V_{j}$ is the set of users

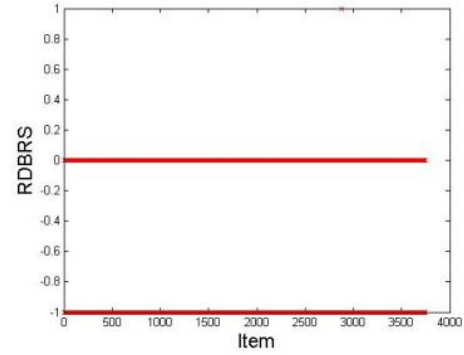

(a) Genuine profile

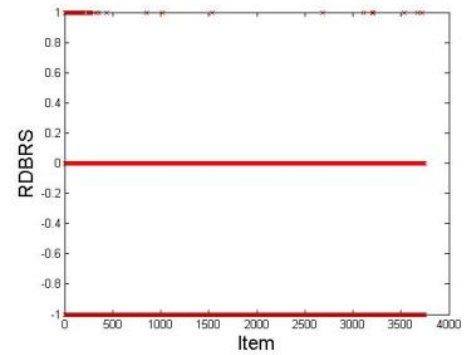

(b) Average attack profile

Fig. 4. Rating Deviation-based rating series. (a) The signal of a genuine profile before DWT; (b) The signal of an average attack profile before DWT

rated by item $j$. If both $V_{i}$ and $V_{j}$ are empty, we define $\operatorname{simi}(\mathrm{i}, \mathrm{j})=0$. Clearly, $0 \leq \operatorname{simi}(\mathrm{i}, \mathrm{j}) \leq 1$.

\section{Novelty-based Rating Series, NBRS}

Let $\mathrm{NoI}_{\mathrm{i}}$ denotes the novelty of item i. Sort all items in set I according to $\mathrm{NoI}_{i}$ in descending order and let $\mathrm{i}=1,2, \ldots,|\mathrm{I}|$ denotes the order of items after sorting. The novelty-based rating series of user $\mathrm{u}, \mathrm{NBRS}_{\mathrm{u}}(\mathrm{i})$ is defined as follows:

$\mathrm{NBRS}_{\mathrm{u}}(\mathrm{i})=$

$\left\{\begin{aligned} 1, & r_{u, i} \neq \perp \text { and }\left(i=1 \text { or } \operatorname{NBRS}_{u}(i-1) \neq 1\right) \\ -1, & r_{u, i}=\perp \text { and }\left(i=1 \text { or } \operatorname{NBRS}_{u}(i-1) \neq-1\right) \\ 0, & \text { otherwise. }\end{aligned}\right.$

where zero value is used to meet the requirements of extreme for DWT [17].

\section{Popularity of Items, PoI}

The popularity of item $\mathrm{i}, \mathrm{PoI}_{i}$, is defined as the number of ratings given to item $\mathrm{i}$ by genuine users in data set [17].

\section{Popularity-based Rating Series, PBRS}

Let $\mathrm{PoI}_{\mathrm{i}}$ denotes the popularity of item i. Sort all items in set I according to $\mathrm{PoI}_{i}$ in descending order and let $\mathrm{i}=1,2, \ldots,|\mathrm{I}|$ denotes the order of items after sorting. The popularity-based rating series of user $\mathrm{u}, \mathrm{PBRS}_{\mathrm{u}}(\mathrm{i})$, is defined as follows:

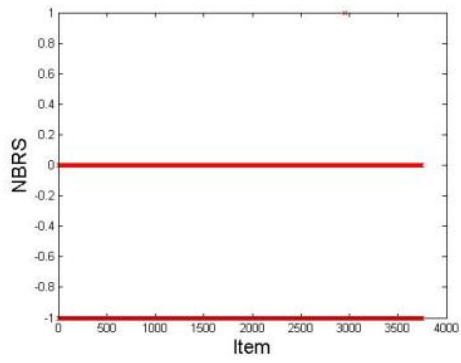

(a) Genuine profile

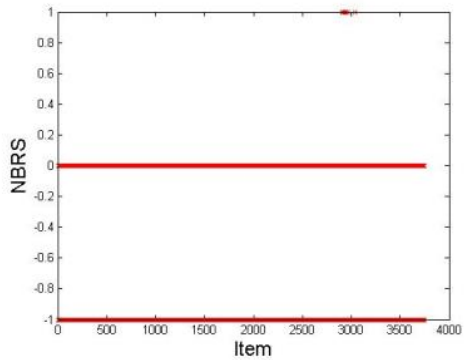

(b) Average attack profile

Fig. 6. Novelty-based rating series. (a) The signal of a genuine profile before DWT; (b) The signal of an average attack profile before DWT
Fig. 5. Popularity-based rating series. (a) The signal of a genuine profile before DWT; (b) The signal of an average attack profile before DWT
$\operatorname{PBRS}_{\mathrm{u}}(\mathrm{i})=$

$\left\{\begin{array}{cl}1, & r_{u, i} \neq \perp \text { and }\left(i=1 \text { or } \operatorname{PBRS}_{u}(i-1) \neq 1\right), \\ -1, & r_{u, i}=\perp \text { and }\left(i=1 \text { or } \operatorname{PBRS}_{u}(i-1) \neq-1\right), \\ 0, & \text { otherwise. }\end{array}\right.$ where zero value is used to meet the requirements of extreme for DWT [17].

To show the difference between genuine and attack profiles in rating series, we give examples of the novelty-based, popularity-based and rating deviation-based rating series in Figures 4-6. These rating series are constructed by the genuine profiles and the average attack profiles (take average attack for example). The genuine profiles are selected from the BookCrossing dataset. As shown in Figures 4-6, there are very little difference between the genuine and average attack profiles in rating series. We can observe that the RDBRS for the genuine profile barely changed from starting position to ending position in compared to the RDBRS of the average attack profile decreased gradually for the rating deviation-based rating series. For the popularity-based rating series, the PBRS for the genuine profile barely changed with the item increased while the PBRS of the average attack profile decreased gradually. And for the novelty-based rating series, the NBRS for genuine profile also almost remain unchanged with the item increased, while the NBRS of the average attack profile show characteristics of more concentrated. As mentioned above, it is 
difficult to discriminate between genuine profiles and attack profiles regardless of using Rating Deviation-based, Popularity-based and Novelty-based rating series. To amplify the difference between genuine profiles and attack profiles, we use DWT to transform the rating series in order to extract features from output signal by using amplitude domain analysis method.

After K (k greater than or equal to 1) level discrete wavelet transform (as shown in Figure 2), we can get the local

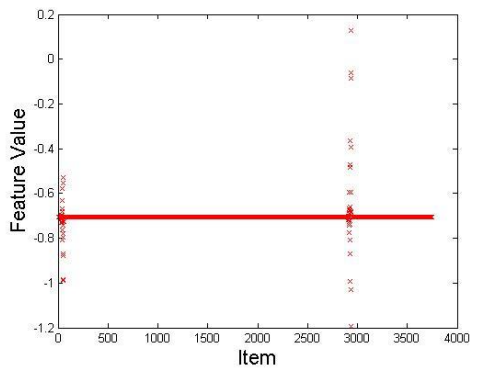

(a) Genuine profile

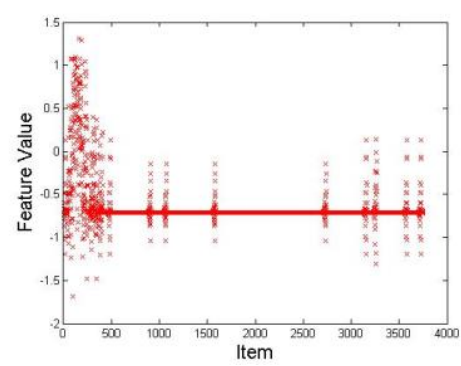

(b) Average attack profile

Fig. 7. The first low-pass output of the rating deviation-based rating series. (a) The signal of a genuine profile after DWT; (b) The signal of an average attack profile after DWT. properties, which passes a series low-pass filters to obtain an approximation coefficients. As shown in Figures 7-9, we can observe that there is a more significant difference between genuine profiles and average attack profiles on rating series than before using DWT. In Figure 7, the strength of oscillations of genuine profiles show characteristics of more concentrated with the item increased while the strength of

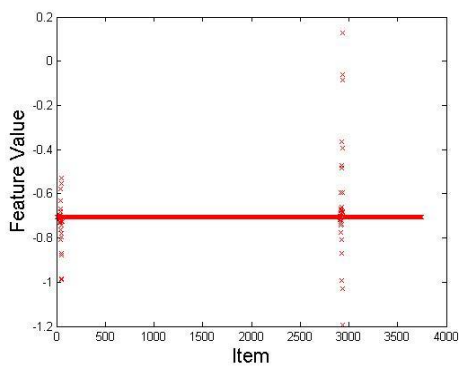

(a) Genuine profile

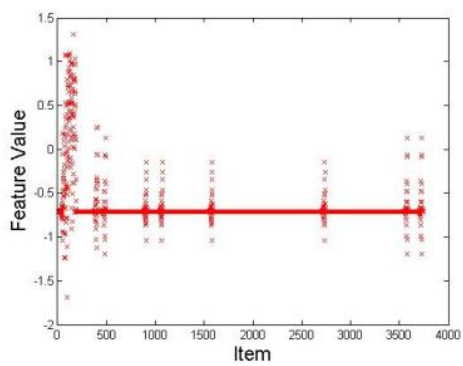

(b) Average attack profile

Fig. 8. The first low-pass output of the popularity-based rating series. (a) The signal of a genuine profile after DWT; (b) The signal of an average attack profile after DWT

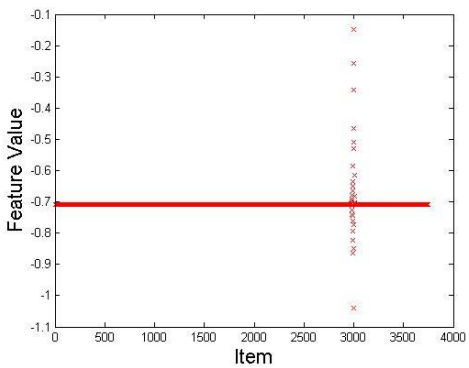

(a) Genuine profiles

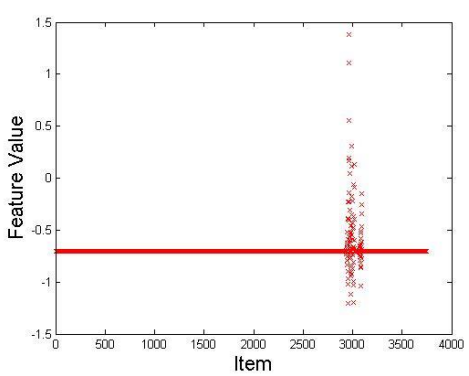

(b) Average attack profiles

Fig. 9. The first low-pass output of the noveltybased rating series. (a) The signal of a genuine profile after DWT; (b) The signal of an average attack profile after DWT

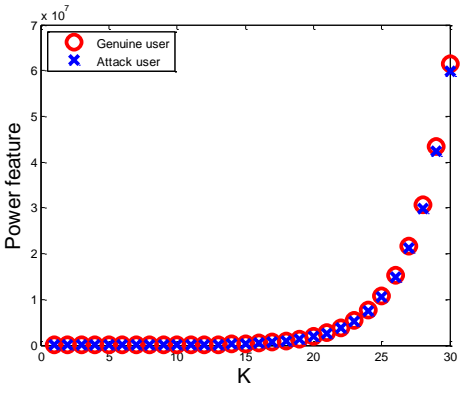

(a)

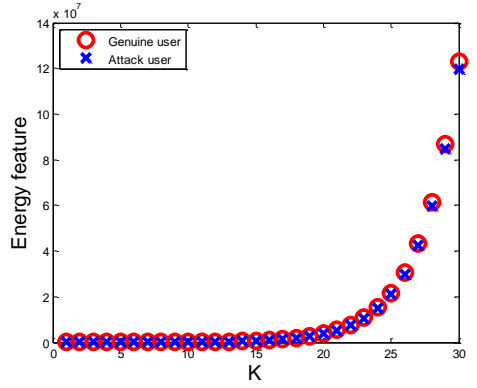

(b)

Fig. 10. The power feature and the energy feature in different $\mathrm{K}$ levels output of discrete wavelet transforms for a genuine user and an attacker. (a) Power features; (b) Energy features

oscillations of average attack profile decreased gradually from starting position to ending position. For the popularity-based rating series, the same observations are also clear in Figure 8. And for the novelty-based rating series, we can observe that there is a little difference between the genuine profiles and average attack profiles, although they show characteristics of more concentrated similarly as illustrated in Figure 9.

Let $F_{-}$RDBRS ${ }_{u}, F_{-} P B R S_{u}$ and $F_{-} N_{B R S}$ denotes the feature vector of user $u$ on the rating deviation-based, novelty-based and popularity-based after DWT, respectively. The proposed feature extraction algorithm is described in algorithm 1. In algorithm 1, from step 1 to step 3 create the rating deviationbased, novelty-based and popularity-based rating series for user u respectively. Step 4 is the process of DWT. Step 5 extract features from approximation parts of rating deviation, popularity and novelty rating series, termed $A_{-} R_{k}, A_{-} P_{k}$ and A_ $\mathrm{N}_{\mathrm{k}}$ by using amplitude domain analysis method. The last step generates a feature space for the stage of detection. 


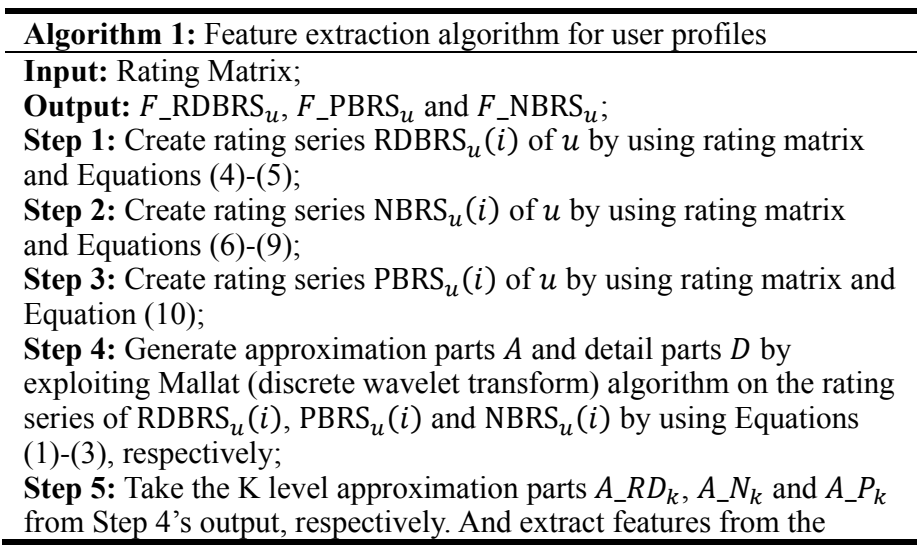

approximation parts by using amplitude domain analysis method on $A_{-} R D_{k}, A_{-} N_{k}$ and $A_{-} P_{k}$ respectively;

Step 6: Generate and return the feature space $F_{-} \mathrm{RDBRS}_{u}, F_{-} \mathrm{PBRS}_{u}$ and $F \_\mathrm{NBRS}_{u}$ respectively.

TABLE III. THE FEATURES OF THE Signal AMPLITUDE DOMAIN AND THEIR DESCRIPTION

\begin{tabular}{|c|c|c|}
\hline Features & Equations & Descriptions \\
\hline Minimum value & $\mathrm{x}_{\min }=\min (\mathrm{X})$ & The minimum value of the amplitude of the signal. \\
\hline Maximum value & $\mathrm{x}_{\max }=\max (\mathrm{X})$ & The maximum value of the amplitude of the signal. \\
\hline Mean value & $\overline{\mathrm{X}}=\operatorname{mean}(\mathrm{X})$ & The average value of the amplitude of the signal. \\
\hline Peak value & $\mathrm{x}_{\mathrm{p}}=\max (\mathrm{abs}(\mathrm{X}))$ & The maximum of the absolute value of the amplitude of the signal. \\
\hline Root mean square value & $\mathrm{X}_{\mathrm{rms}}=\sqrt{\frac{1}{\mathrm{~N}} \sum_{\mathrm{i}=1}^{\mathrm{N}} \mathrm{x}_{\mathrm{i}}^{2}}$ & The root mean square value of the amplitude of the signal. \\
\hline Root mean square amplitude value & $\mathrm{X}_{\mathrm{r}}=\left(\frac{1}{\mathrm{~N}} \sum_{\mathrm{i}=1}^{\mathrm{N}} \sqrt{\left|\mathrm{x}_{\mathrm{i}}\right|}\right)^{2}$ & Represent the energy size of the signal. \\
\hline Absolute mean & $|\bar{X}|=\frac{1}{N} \sum_{i=1}\left|x_{i}\right|$ & Absolute mean value of the amplitude of the signal. \\
\hline Variance & $\sigma_{\mathrm{x}}^{2}=\mathrm{X}_{\mathrm{rms}}^{2}-\overline{\mathrm{X}}^{2}$ & Represent the degree of dispersion of the signal. \\
\hline Skewness & $\alpha=\frac{1}{N} \sum_{i=1}^{N} x_{i}^{3}$ & $\begin{array}{l}\text { Represent the asymmetry of amplitude probability density function on } \\
\text { the vertical axis. }\end{array}$ \\
\hline Kurtosis & $\beta=\frac{1}{N} \sum_{i=1}^{N} x_{i}^{4}$ & Represent the steep degree of the signal curve. \\
\hline Shape factor & $\mathrm{S}_{\mathrm{f}}=\mathrm{X}_{\mathrm{rms}} /|\overline{\mathrm{X}}|$ & $\begin{array}{l}\text { A shape factor refers to a value that is affected by an object's shape but } \\
\text { is independent of its dimensions }\end{array}$ \\
\hline Crest factor & $\mathrm{C}_{\mathrm{f}}=\mathrm{X}_{\max } / \mathrm{X}_{\mathrm{rms}}$ & $\begin{array}{l}\text { Crest factor is a measure of a waveform, showing the ratio of peak } \\
\text { values to the average value. }\end{array}$ \\
\hline Impulse factor & $\mathrm{I}_{\mathrm{f}}=\mathrm{X}_{\max } /|\overline{\mathrm{X}}|$ & Non-dimensional parameter in amplitude domain. \\
\hline Clearance factor & $\mathrm{CL}_{\mathrm{f}}=\mathrm{X}_{\max } / \mathrm{X}_{\mathrm{r}}$ & Non-dimensional parameter in amplitude domain. \\
\hline Kurtosis value & $\mathrm{K}_{\mathrm{v}}=\beta / \mathrm{X}_{\mathrm{rms}}^{4}$ & Non-dimensional parameter in amplitude domain. \\
\hline
\end{tabular}

For different types of signal, there are different analysis methods such as time domain analysis, frequency domain analysis and amplitude domain analysis. As shown in Figure 10 , we can observe that these are no significant difference between genuine user and attacker with the $\mathrm{K}$ (the $\mathrm{K}$ level output of DWT) increased, regardless of using the power features or energy features. In this paper, we use amplitude domain analysis to extract features from signals. The details of signal features in amplitude domain are showed in Table 3. We have 15 features to characterize the signal which extracts from the $\mathrm{K}$ level (we set K equal to 1 in our work) output of DWT.

Algorithm 2: Detection algorithm

Input: The set of users' feature space $F_{-} \mathrm{RDBRS}_{u}, F_{-} \mathrm{PBRS}_{u}$ and $F_{-} \mathrm{NBRS}_{u}$; The number of clusters $k$;

Output: The detected result $D$;

Step 1: $\left\{C_{-} \mathrm{RD}_{1}, C_{-} \mathrm{RD}_{2}\right\} \leftarrow \operatorname{EM}\left(F_{-} \mathrm{RDBRS}_{u}\right)$;
Step 2: $\left\{C_{-} \mathrm{P}_{1}, C_{-} \mathrm{P}_{2}\right\} \leftarrow \operatorname{EM}\left(F_{-} \mathrm{PBRS}_{u}\right)$;

Step 3: $\left\{C_{-} \mathrm{N}_{1}, C_{-} \mathrm{N}_{2}\right\} \leftarrow \operatorname{EM}\left(F_{-} \mathrm{NBRS}_{u}\right)$;

Step 4: $D_{-} A R D=\min \left(C_{-} \mathrm{RD}_{1}, C_{-} \mathrm{RD}_{2}\right), D_{-} \mathrm{P}=\min \left(C_{-} \mathrm{P}_{1}, C_{-} \mathrm{P}_{2}\right)$,

$D_{-} \mathrm{N}=\min \left(C_{-} \mathrm{N}_{1}, C_{-} \mathrm{N}_{2}\right)$;

Step 5: $D \leftarrow\left\{D \mid D \_\mathrm{RD} \cap D_{-} \mathrm{P} \cap D_{-} \mathrm{N}\right\}$;

Return $D$.

\section{Detection algorithm}

In order to get better detection performance as far as possible, we combine the rating deviation-based, novelty-based and popularity-based methods to distinguish between genuine profiles and attack profiles. And then, we utilize EM (Expectation-maximization) clustering method (Clustering results and EM clustering method were created using Weka ${ }^{3}$ ) to separate attackers from genuine users as far as possible. Let

\footnotetext{
${ }^{3}$ http://www.cs.waikato.ac.nz/ml/weka/
} 
D denotes the set of detection result. The proposed method for detecting grey attacks is described in algorithm 2. In algorithm

2, from step 1 to 3 perform EM algorithm on feature vector $\mathrm{F}_{-} \mathrm{RDBRS}_{\mathrm{u}}, \mathrm{F}_{-} \mathrm{PBRS}_{\mathrm{u}}$ and $\mathrm{F}_{-} \mathrm{NBRS}_{\mathrm{u}}$, respectively. Step 4 obtains the set of attackers decided by using the smaller cluster, since the number of attackers less than the number of genuine users in the recommender system. In step 5, we exploit the intersection of the set $\mathrm{D}_{-} \mathrm{RD}$, D_P and D_N, and then the detection result D was generated.

\section{EXPERIMENTS AND ANALYSIS}

In this section, we firstly show the experimental data and settings on a real-world dataset. Then, we discuss our experimental results.

\section{A. Experimental data and settings}

In our experiments, we use the Book-Crossing ${ }^{4}$ dataset. It contains 278,858 users providing $1,149,780$ ratings (explicit or implicit) about 271,379 books and each rater had to rate at least 1 books. All ratings are in the form of integral values between minimum value 1 and maximum value 10. The minimum score means the rater dislikes the book, while the maximum score means the rater enjoyed the book. We randomly select 800 genuine profiles from the dataset as the samples of genuine profiles. For the attack profiles, we just focus on nuke attacks

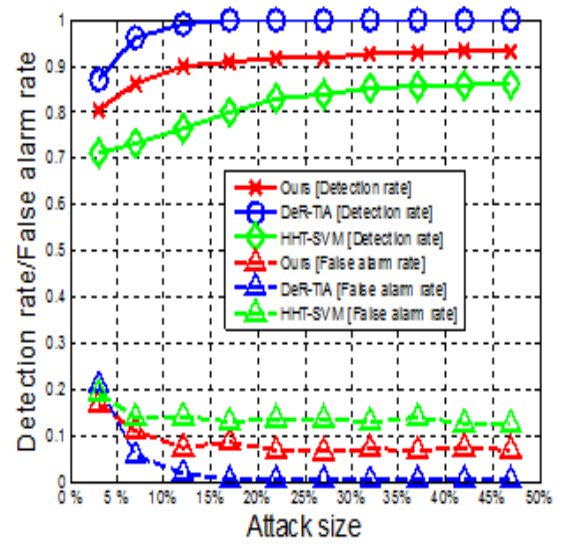

(a)

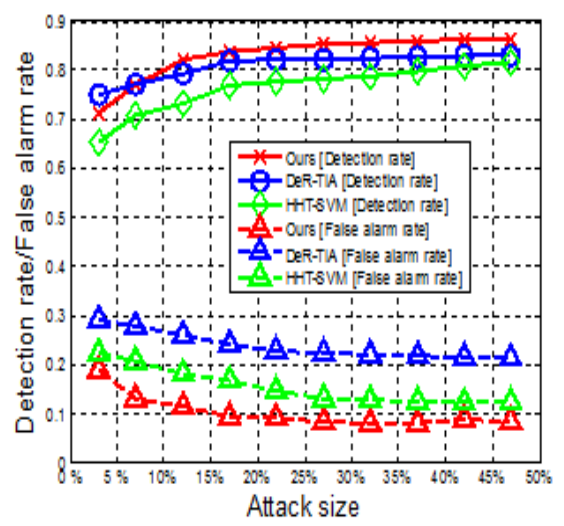

(b)

Fig. 11. The comparison of detection rate and false alarm rate in different attack sizes. (a) Grey rating is 1 , filler size is $5 \%$, single-target bandwagon (random) attack; (b) Grey rating is 3, filler size is $5 \%$, singletarget bandwagon (random) attack

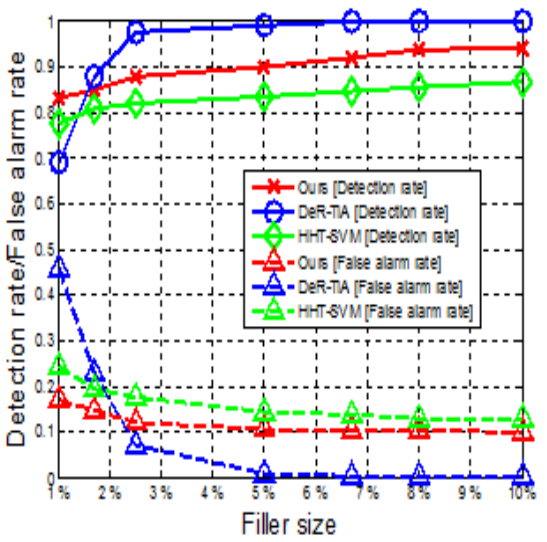

(a)

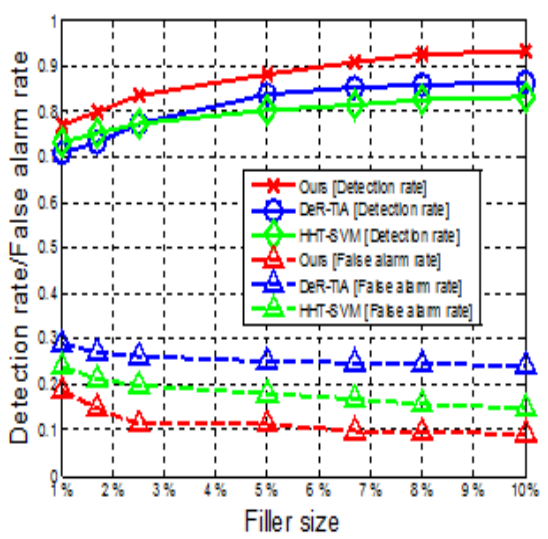

(b)

Fig. 12. The comparison of detection rate and false Fig. 13. The comparison of detection rate and false alarm rate in different filler sizes. (a) Grey rating is 1, alarm rate with different grey ratings in single-target attack size is $17 \%$, single-target bandwagon (random) attack. (a) Filler size is $5 \%$, attack size varies in attack; (b) Grey rating is 3, attack size is 17\%, single- bandwagon (average) attack. (b) Attack size is 17\%, target bandwagon (random) attack

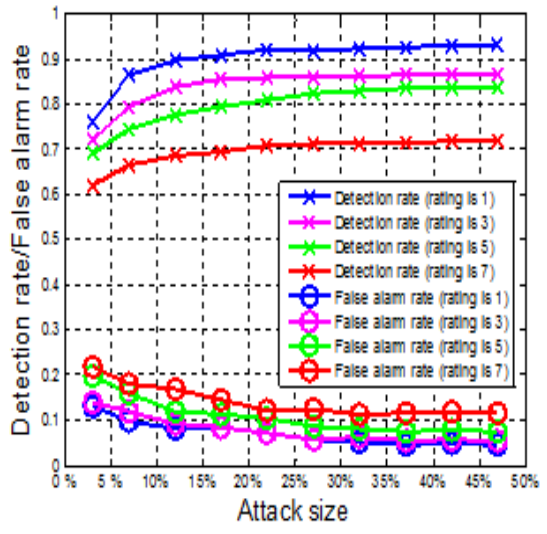

(a)

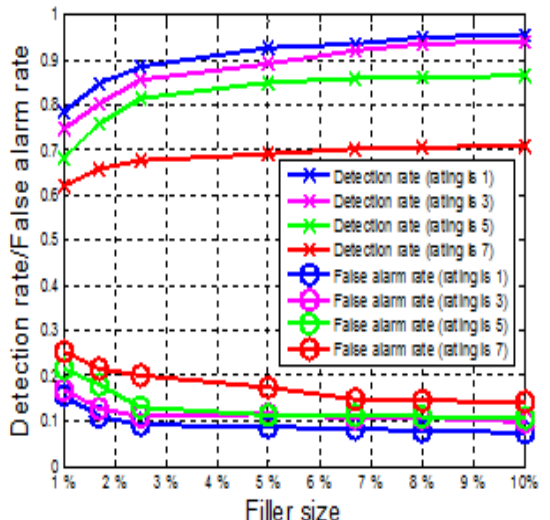

(b) filler size varies in bandwagon (average) attack 
TABLE IV. COMParison of the Detection PERformance of OUR Method with Two Benchmarked Methods

\begin{tabular}{|c|c|c|c|c|c|c|c|c|c|}
\hline \multirow{3}{*}{ Attack models } & \multirow{3}{*}{ Methods } & \multicolumn{8}{|c|}{ Rating } \\
\hline & & \multicolumn{2}{|c|}{1} & \multicolumn{2}{|c|}{3} & \multicolumn{2}{|c|}{5} & \multicolumn{2}{|c|}{7} \\
\hline & & $D R$ & FAR & $D R$ & FAR & $D R$ & FAR & $D R$ & FAR \\
\hline \multirow{3}{*}{ AOP } & HHT-SVM & 0.845 & 0.095 & 0.819 & 0.15 & 0.79 & 0.177 & 0.673 & 0.21 \\
\hline & DeR-TIA & 1.0 & 0.005 & 0.715 & 0.185 & 0.734 & 0.225 & 0.707 & 0.275 \\
\hline & Ours & 0.911 & 0.0785 & 0.835 & 0.093 & 0.813 & 0.102 & 0.702 & 0.11 \\
\hline \multirow{3}{*}{ Random } & HHT-SVM & 0.819 & 0.12 & 0.765 & 0.15 & 0.7345 & 0.14 & 0.68 & 0.21 \\
\hline & DeR-TIA & 1.0 & 0.0025 & 0.735 & 0.175 & 0.727 & 0.195 & 0.731 & 0.265 \\
\hline & Ours & 0.904 & 0.081 & 0.834 & 0.086 & 0.801 & 0.093 & 0.707 & 0.11 \\
\hline \multirow{3}{*}{ Average } & HHT-SVM & 0.873 & 0.1091 & 0.782 & 0.13 & 0.759 & 0.158 & 0.665 & 0.182 \\
\hline & DeR-TIA & 1.0 & 0.0025 & 0.763 & 0.165 & 0.750 & 0.205 & 0.752 & 0.195 \\
\hline & Ours & 0.907 & 0.085 & 0.837 & 0.090 & 0.805 & 0.079 & 0.703 & 0.125 \\
\hline \multirow{3}{*}{ Bandwagon (average) } & HHT-SVM & 0.906 & 0.09 & 0.8279 & 0.14 & 0.7869 & 0.16 & 0.675 & 0.19 \\
\hline & DeR-TIA & 1.0 & 0.005 & 0.755 & 0.18 & 0.734 & 0.25 & 0.752 & 0.285 \\
\hline & Ours & 0.935 & 0.0615 & 0.852 & 0.0713 & 0.823 & 0.0682 & 0.705 & 0.115 \\
\hline \multirow{3}{*}{ Bandwagon (random) } & HHT-SVM & 0.910 & 0.095 & 0.8179 & 0.13 & 0.8069 & 0.18 & 0.67 & 0.21 \\
\hline & DeR-TIA & 1.0 & 0.005 & 0.747 & 0.165 & 0.735 & 0.205 & 0.750 & 0.27 \\
\hline & Ours & 0.934 & 0.055 & 0.868 & 0.075 & 0.83 & 0.069 & 0.718 & 0.115 \\
\hline \multirow{3}{*}{ Segment } & HHT-SVM & 0.897 & 0.0891 & 0.819 & 0.13 & 0.7869 & 0.167 & 0.667 & 0.193 \\
\hline & DeR-TIA & 1.0 & 0.0055 & 0.752 & 0.15 & 0.730 & 0.185 & 0.731 & 0.25 \\
\hline & Ours & 0.915 & 0.075 & 0.846 & 0.08 & 0.815 & 0.086 & 0.70 & 0.11 \\
\hline \multirow{3}{*}{ Reveres bandwagon } & HHT-SVM & 0.895 & 0.087 & 0.8179 & 0.125 & 0.796 & 0.145 & 0.66 & 0.195 \\
\hline & DeR-TIA & 1.0 & 0.005 & 0.739 & 0.175 & 0.754 & 0.185 & 0.727 & 0.26 \\
\hline & Ours & 0.933 & 0.065 & 0.868 & 0.075 & 0.815 & 0.0775 & 0.705 & 0.125 \\
\hline \multirow{3}{*}{ Love/Hate } & HHT-SVM & 0.849 & 0.105 & 0.807 & 0.135 & 0.7569 & 0.175 & 0.67 & 0.205 \\
\hline & DeR-TIA & 1.0 & 0.0025 & 0.752 & 0.16 & 0.727 & 0.195 & 0.750 & 0.24 \\
\hline & Ours & 0.917 & 0.075 & 0.845 & 0.065 & 0.81 & 0.0785 & 0.717 & 0.135 \\
\hline
\end{tabular}

and their grey attacks, push attacks can be detected in the analogous manner. For each attack model (as shown in Table 2 ), we respectively generate nuke and grey attack profiles according to the corresponding attack models with diverse attack sizes ${ }^{5}\{3 \%, 7 \%, 12 \%, 17 \%, 22 \%, 27 \%, 32 \%, 37 \%$, $42 \%, 47 \%\}$ and filler sizes ${ }^{6}\{1 \%, 1.7 \%, 2.5 \%, 5 \%, 6.7 \%, 8 \%$, $10 \%\}$. In addition, to ensure the rationality of the results, the target item is randomly selected for these attack profiles. Especially in Table 2, the $r_{\text {grey }}$ is the grey rating on target items rated by lower scores such as 1, 3, 5 and 7 .

The generated attack profiles are respectively inserted into the sampled genuine profiles to construct our test datasets. Therefore, we have $560(8 \times 10 \times 7)$ test datasets including 8 diverse attack models, 10 different attack sizes and 7 different filler sizes. Notice that, these process is repeated 10 times and the average value of detection results are reported for the experiments. All numerical studies are implemented using MATLAB R2012a on a personal computer with Intel(R) Core(TM) i7-4790 3.60GHz CPU, 16G memory and Microsoft windows 7 operating system.

To measure detection performance of the proposed methods, we use detection rate and false alarm rate in our experiments.

$$
\begin{aligned}
\text { detection rate } & =\frac{|\mathrm{D} \cap \mathrm{A}|}{|\mathrm{A}|} \\
\text { false alarm rate } & =\frac{|\mathrm{D} \cap \mathrm{G}|}{|\mathrm{G}|}
\end{aligned}
$$

\footnotetext{
${ }^{5}$ The ratio between the number of attackers and genuine users.

${ }^{6}$ The ratio between the number of items rated by user $u$ and the number of entire items in the recommender systems.
}

where D is the set of the detected user profiles, $A$ is the set of attacker profiles, and $\mathrm{G}$ is the set of genuine user profiles [11].

\section{B. Experimental results and analysis}

To validate the detection performance of our proposed method, we employ two benchmarked methods including HHT-SVM [17] and DeR-TIA [1] to demonstrate the outperformance of our method. Take bandwagon (random) attack for example, Figures 11 and 12 demonstrate how each method performs under varying attack sizes and filler sizes, respectively. In the bandwagon (random) attack, a group isolated attackers always provide maximal or minimal or grey rating on a set of items when they are selected as the selected items or the filler items. As shown in Figures 11(a) and 12(a), the detection rate increased gradually and false alarm rate decreased gradually when the attack size increased and the filler size is fixed with 5\% (in Figure 11 (a)) and filler size increased and attack size is $17 \%$ (in Figure 12 (a)). In addition, we can observe that our method shows significantly better detection performance than HHT-SVM with the attack size increased. This might be attributed to the combination of novelty-based, popularity-based and rating deviation-based rating series adopted by our proposed algorithm. The rating deviation-based strategy calculates a rating offset on a target item which can identify between the genuine profiles and attack profiles. The second observation is that DeR-TIA shows the best performance among the three algorithms. With the attack size increasing, the detection rate almost keeps maximum $100 \%$ and the false alarm rate almost keeps minimum 0, except for the early stages (attack size $<17 \%$ ) as illustrated in Figure 11 (a). The same observations are also clear in Figure 12(a). However, for grey rating, as shown in Figures 11 (b) and 12 (b), we set a grey rating equal to 3 (integer rating from 1-10 in the datasets). Our method shows the best detection performance among the three methods, 
although the detection rate of our method shows lower than DeR-TIA in the early stage (attack size $<12 \%$ ) as illustrated in Figure 11 (b). To compare with our proposed method and HHT-SVM, DeR-TIA shows higher false alarm rate than the others. Moreover, the detection rate of DeR-TIA almost remained unchanged with the attack size increased, and similar results can be observed in Figure 12 (b). The results might be attributed to grey rating. The first phase of DeR-TIA can filter out a part of genuine users by using similarity threshold, but it is difficult to capture the suspected profiles which rate grey ratings in their second phase. They defend and remove the suspected users almost depend on the similarity threshold, so they perform lower detection performance. For our proposed method, we pay more attention to the details of the all ratings rated by a user and explore the top-N items which has sorted by the rating deviation of item in order to characterize the grey ratings.

To examine the detection performance of our method in bandwagon (random) attack with different grey ratings (take bandwagon (random) attack for example), we conduct a list of experiments with diverse attack sizes and filler sizes. As shown in Figure 13, we perform 4 different grey ratings including 1, 3, 5 and 7 on the target items. One observation is that the detection rate gradually increased and false alarm rate gradually decreased with the attack size increasing (in Figure 13 (a)) or filler size increasing (in Figure 13 (b)). The other observation is that the detection performance gradually performs poor when the grey rating increased from 1 to 7 , regardless of different attack sizes and filler sizes. The results may indicate that the grey ratings are close to average rating in the entire system with the grey rating on the target items increasing. The attackers rate a mean rating may show a rating behavior like genuine users, which is difficult to discriminate between attackers and genuine users and shows higher false alarm rate.

To further illustrate the detection performance of our proposed method under different attack models with different grey ratings, we conduct a list of experiments in 8 attack models for comparing the performance of our proposed method with HHT-SVM and DeR-TIA. We use 4 different ratings including 1, 3, 5 and 7 score when filler size is $5 \%$ and attack size is $17 \%$. As shown in Table 4 , we can observe that the detection rate (DR) of our method reports higher than other two benchmarked methods when the grey rating increasing, except for the grey rating is 1 . Similarly, the false alarm rate (FAR) of our method reports lower than others. In addition, the second observation is that the proposed method reports better detection performance under bandwagon (both random and average) and reverse bandwagon attacks in comparison with the other attack models, especially for grey ratings (such as 3, 5 and 7 score). These results may indicate that we combine the rating deviation-based, novelty-based and popularity-based rating series in our method is useful to discriminate difference between grey attack profiles and genuine profiles. The rating deviation-based rating series may easily characterize the grey attacks in comparison with the other two methods.

\section{CONCLUSIONS AND FUTURE WORK}

In this paper, we highlighted the challenges faced by the grey attacks, and then we develop an unsupervised detection approach based on discrete wavelet transform by combing the rating deviation-based, novelty-based and popularity-based rating series. Extensive experiments on the Book-Crossing dataset have demonstrated the effectiveness of the proposed approach. One of the limitations of our proposed method directly comes from the time consumption, which constructs the signals of rating series. In our future work, we intend to extend and improve grey attack detection in the following directions: 1) Considering more attack models such as Power users attack or Power items attack, etc.; 2) We will explore specific and simple method to detect grey attacks and develop better approach to construct the rating series. 3) Extracting more simpler and effective features to characterize grey attack profiles is still an open issue.

\section{ACKNOWLEDGMENT}

The research is supported by NFSC $(61175039,61221063)$, 863 High Tech Development Plan (2012AA011003), Research Fund for Doctoral Program of Higher Education of China (20090201120032), International Research Collaboration Project of Shaanxi Province (2013KW11) and Fundamental Research Funds for Central Universities (2012jdhz08).

\section{REFERENCES}

[1] W Zhou, Y. S. Koh, J. H. Wen, S Burki and G Dobbie. Detection of abnormal profiles on group attacks in recommender systems. Proceedings of the 37th international ACM SIGIR conference on Research \& development in information retrieval, Pages 955-958, 2014.

[2] D. Jia, F. Zhang and S. Liu. A robust collaborative filtering recommendation algorithm based on multidimensional trust model. Journal of Software, vol. 8, no. 1, 2013.

[3] R. Burke, B. Mobasher and C. Williams. Classification features for attack detection in collaborative recommender systems. In Proceedings of the 12th International Conference on Knowledge Discovery and Data Mining, pages 17-20, 2006.

[4] B. Mobasher, R. Burke and J. Sandvig. Model-based collaborative filtering as a defense against profile injection attacks. AAAI. 1388, 2006.

[5] K. Bryan, M. O’Mahony and P. Cunningham. Unsupervised retrieval of attack profiles in collaborative recommender systems. In RecSys'08: Proceedings of the 2008 ACM conference on Recommender systems, pages $155-162,2008$.

[6] H. Hurley, Z. Cheng and M. Zhang. Statistical attack detection. In: Proceedings of the Third ACM Conference on Recommender Systems (RecSys'09), pages 149-156, 2009.

[7] B. Mehta. Unsupervised shilling detection for collaborative filtering. AAAI, 1402-1407, 2007.

[8] C Li and Z Luo. Detection of shilling attacks in collaborative filtering recommender systems. In: Proceedings of the international conference of soft computing and pattern recognition, Dalian, China, pages 190$193,2011$.

[9] I Gunes, C Kaleli, A Bilge and H Polat. Shilling attacks against recommender systems: A comprehensive survey. Artificial Intelligence Review, pages 1-33, 2012.

[10] N Giseop, Y. Kang and C. Kim. Ecsy-Recsy: Considering Sybil attack with time dynamics and economics in recommender system. International Conference on Information Networking (ICOIN), pages $566-571,2013$. 
[11] C. Chung, P. Hsu and S. Huang. $\beta$ P: A novel approach to filter out malicious rating profiles from recommender systems. Journal of Decision Support Systems, pages 314-325, April 2013.

[12] X. Zhang, T. Lee and G Pitsilis. Securing recommender systems against shilling attacks using social-based clustering. Journal of Computer Science and Technology (JCST), pages 616-624, July 2013.

[13] Z Zhang and S. Kulkarni. Graph-based detection of shilling attacks in recommender systems. IEEE International Workshop on Machine Learning for Signal Processing (MLSP), pages 1-6, 2013.

[14] B. Mehta, T. Hofmann and P. Fankhauser. Lies and propaganda: detecting spam users in collaborative filtering. In: IUI '07: Proceedings of the 12th International Conference on Intelligent User Interfaces, pages 14-21, 2007.

[15] M Morid and M Shajari. Defending recommender systems by influence analysis. Information Retrieval, pages 137-152, April 2014.

[16] Z. Wu, J Cao, B Mao and Y. Zhang. Semi-SAD: Applying semisupervised learning to shilling attack detection. Proceedings of the 5th International Conference on Recommender Systems. New York: ACM, pages 289-292, 2011.

[17] F. Zhang and Q. Zhou. HHT-SVM: An online method for detecting profile injection attacks in collaborative recommender systems, Knowl. Based Syst. 2014.

[18] J Zou and F Fekri. A belief propagation approach for detecting shilling attacks in collaborative filtering. Proceedings of the 22nd ACM international conference on Conference on information \& knowledge management (CIKM), pages 1837-1840, 2013.

[19] Z Zhang and SR Kulkarni, Detection of Shilling Attacks in Recommender Systems via Spectral Clustering. 2014 17th International Conference on Information Fusion (FUSION). Page(s):1-8, 7-10 July 2014.

[20] Fidel Cacheda, Victor Carneiro, Diego Fernandez and vreixo Formoso. Comparison of Collaborative Filtering Algorithms: Limitations of Current Techniques and Proposals for Scalable, High-Performance Recommender Systems. ACM Transactions on the Web (TWEB), Volume 5, Issue 1, February 2011.

[21] B. Mobasher, R. Burke, B. Bhaumil and C. Williams. Towards trustworthy recommender systems: an analysis of attack models and algorithm robustness. ACM Transactions on Internet Technology, 7 (4), pages 23-38, 2007.
[22] C. E. Seminario and D. C. Wilson. Attacking item-based recommender systems with power items. RecSys'14, October 6-10, 2014.

[23] M. J. Shensa, Wedding the a trous and Mallat algorithms, IEEE Trans. Signal Process. 40 (1992), 24642482.

[24] Williams, C., Mobasher, B., Burke, R., Sandvig, J., Bhaumik, R. Detection of obfuscated attacks in collaborative recommender systems. In: Workshop on Recommender Systems, ECAI, 2006.

[25] J. S. Lee, D. Zhu, Shilling attack detection: a new approach for a trustworthy recommender system, JNFORMS J. Comput. 24 (1) , pages 117-131, 2011.

[26] B. Mehta, W. Nejdl, Unsupervised strategies for shilling detection and robust collaborative filtering, User Model. User-Adap. Inter. 19 (1-2), pages 65-79, 2009.

[27] Mohamed Hamdi, Noureddine Boudriga. Detecting denial-of-service attacks using the wavelet transform. Computer Communications, 30 (16) (2007), pp. 3203-3213.

[28] C.A. Williams, B. Mobasher, R. Burke, R. Bhaumik, Detecting profile injection attacks in collaborative filtering: a classification-based approach, in: Proceedings of the 8th Knowledge Discovery on the Web International Conference on Advances in Web Mining and Web Usage Analysis (Lecture Notes in Computer Science), Springer-Verlag, 2007, pp. $167-186$

[29] B. Mobasher, R. Burke, R. Bhaumik, and C. Williams, "Toward trustworthy recommender systems: An analysis of attack models and algorithm robustness," ACM Transactions on Internet Technology (TOIT), Volume 7 , Issue 4 (October 2007), 2007.

[30] Z. A. Wu, J. J. Wu, J. Cao, D. C. Tao, HySAD: a semi-supervised hybrid shilling attack detector for trustworthy product recommendation, in: 18th ACM SIGKDD Conference on Knowledge Discovery and Data Mining, Beijing, China, August, 2012, pp. 985-993.

[31] F. Zhang, Q. Zhou, A meta-learning-based approach for detecting profile injection attacks in collaborative recommender systems, J. Comput. 7 (1) (2012) 226-234.

[32] F. He, X.Wang, B. Liu, Attack detection by rough set theory in recommendation system, in: Proceedings of 2010 IEEE International Conference on Granular Computing, 2010, pp. 692-695.

[33] W. Zhou, J. Wen, Y. S. Koh, Q. Xiong, M. Gao, G. Dobbie, and S. Alam. Shilling attacks detection in recommender systems based on target item analysis. PloS one, 2015. 